\title{
Catastrophic Antiphospholipid Antibody Syndrome in Systemic Lupus Erythematosus: An Autopsy Case Report of a Young Woman
}

\author{
Reiko Mizuno, Shinichi Fujimoto*, Takashi Fujimoto*, Toshihiko Nishino, Hideo SHIIkI, \\ Toshio Hashimoto, Shinobu NaKamURA* and Kazuhiko DoHI
}

\begin{abstract}
Catastrophic antiphospholipid syndrome (CAPS) is a severe variant of antiphospholipid syndrome (APS) characterized by disseminated microangiopathy that results in multiorgan failure. CAPS mainly occurs in association with systemic lupus erythematosus (SLE). Clinically, CAPS mimics disseminated SLE vasculitis, intravascular coagulation (DIC), and particularly thrombotic thrombocytopenic purpura (TTP). We describe an autopsy case of young woman with CAPS in SLE, which is difficult to differentiate from TTP secondary to SLE. (Internal Medicine 39: 856-859, 2000)
\end{abstract}

Key words: anticardiolipin antibodies (aPL), thrombotic thrombocytopenic purpura (TTP)

\section{Intoroduction}

Approximately $30 \%$ of patients with systemic lupus erythematosus (SLE) has high levels of antiphospholipid antibodies (aPL), namely anticardiolipin antibodies (aCL) or lupus anticoagulant (LAC) (1). The presence of these is associated with an increased incidence of arterial and venous thrombosis ranging from 11-100\% (2). Love and Santoro estimated an overall incidence of thrombotic complication of $25 \%$ in their LAC series and $28 \%$ in their aCL series (2). In 1987, Harris et al first proposed criteria for antiphospholipid syndrome (APS) which included the presence of aPL in combination with arterial, venous, or placental thrombosis and thrombocytopenia (3). APS may be primary or secondary to some systemic disease, especially SLE. In 1992, Asherson first described catastrophic APS (CAPS) which was a severe variant of APS characterized by disseminated microangiopathy that results in multiorgan failure (4). CAPS mainly occurs in association with SLE. Clini- cally, CAPS may exactly mimic SLE vasculitis, disseminated intravascular coagulation (DIC), and particularly thrombotic thrombocytopenic purpura (TTP). Therefore, the differential diagnosis in patients with CAPS in SLE is often difficult. We describe an autopsy case of CAPS in SLE and discuss its differential diagnosis.

For editorial comment, see $\mathrm{p} 763$.

\section{Case Report}

A 28-year-old woman was transferred to our hospital because of progressive renal failure on December 30, 1996. She had been diagnosed with idiopathic thrombocytopenic purpura (ITP) at the age of 9, based on thrombocytopenia and the presence of antiplatelet antibodies. No significant family history was obtained, except that her parent's marriage was consanguineous. She was treated with $10 \mathrm{mg} /$ day of prednisolone. Two years later, at age 11, she was diagnosed with SLE, based on the presence of a malar rash, polyarthritis, high titers of antinuclear antibodies (ANA) (1:320 with speckled pattern) and anti-double stranded (ds) DNA antibodies (48 IU/ml), at that time aPL analysis was not performed. When she was 19 years old she developed autoimmune hemolytic anemia (AIHA). Two years later, at age 21, she began to manifest mild, but various neurologic abnormalities including muscle weakness, sensory impairment, and the feeling of numbness in the extremities, spastic gait, and dysarthria. Magnetic resonance imaging (MRI) of the brain revealed atrophy of bilateral frontal and parietal lobes. Concurrently she demonstrated severe thrombocytopenia and high levels of aCL (IgG class) (120 U/ $\mathrm{ml}$; normal value $<20 \mathrm{U} / \mathrm{ml}$ ), which was first performed this time. Anticoagulation therapy was started. In addition, courses of pulse therapy with methylprednisolone and intravenous cyclophosphamide therapy were carried out, but these therapies had little effect on thrombocytopenia. Neurologic dysfunction

From the First Department of Internal Medicine and *the Department of General Medicine and Clinical Investigation, Nara Medical University, Nara Received for publication November 9, 1999; Accepted for publication March 7, 2000

Reprint requests should be addressed to Dr. Reike Mizuno, the First Department of Internal Medicine, 840 Shijo Kashihara Nara 634-8522 
had been stable. Three weeks before the transfer to our institution she was admitted to another hospital because of development of pneumonia. On the admission, mild neurologic dysfunction was seen but she could walk and had been well oriented. Renal function was normal. The disease activity of SLE itself was well controlled. The pneumonia had improved with antibiotics. But one week after improvement of pneumonia, she developed progressive renal failure (serum concentration of creatinine; $2.9 \mathrm{mg} / \mathrm{dl}$ ). Also neurologic dysfunction was rapidly exacerbated. She became drowsy and poorly oriented. She could not walk by herself and presented involuntary movement. She was sent to our hospital. On physical examination, her temperature was $39.1^{\circ} \mathrm{C}$; pulse, regular at $120 / \mathrm{min}$; and blood pressure, $180 / 88 \mathrm{mmHg}$. She was drowsy and poorly oriented. Her conjuctivae were pale and icteric. Her face was puffy and pretibial edema was noted. Fine crackles were slightly heard at the bases of both lungs. The abdomen was distended and revealed shifting dullness. Multiple purpuric lesions were seen on the extremities. On neurologic examination, in addition to paresis in the extremities, involuntary movement, hypesthesia, and dysarthria were present. Urinalysis revealed proteinuria (4.6 g/day) and hematuria (3+). Laboratory investigation disclosed normocytic anemia (hemoglobin, $4.7 \mathrm{~g} / \mathrm{dl}$ ) with $14 \%$ reticulocytes, thrombocytopenia (platelet count, 36,000/ $\mathrm{mm}^{3}$ ), and elevated serum concentrations of urea nitrogen (26 $\mathrm{mg} / \mathrm{dl})$, creatinine $(2.9 \mathrm{mg} / \mathrm{dl})$, total bilirubin $(2.1 \mathrm{mg} / \mathrm{dl})$, and lactate dehydrogenase $(2,079 \mathrm{IU} / l)$. The partial thromboplastin time, prothrombin time, and fibrinogen concentration were normal. Haptoglobin was undetectable. The $\mathrm{C} 3$ and $\mathrm{C} 4 \mathrm{com}-$ ponents all were slightly depressed. Biologically, a false-positive reaction was seen. Direct and indirect Coombs tests were positive. The ANA test was positive at a titer of 1:40 with a speckled pattern. Anti-ds DNA antibodies were not detected. A high level of aCL (IgG class) was demonstrated (124.5 U/ $\mathrm{ml}$ ). The lupus anticoagulant (phospholipid neutralization assay; 14.9 seconds) and antiplatelet antibodies (enzyme-linked immunosorbent assay; $38 \mathrm{ng} / 10^{7}$ platelets) were increased. A peripheral blood smear demonstrated numerous fragmented red cells and many schistocytes. Blood cultures yielded no growth. MRI of the brain revealed severe global atrophy and infarct area in the occipital lobe (Fig. 1). Methylprednisolone pulse therapy, intravenous immunoglobulin and cyclophosphamide, in addition plasmapheresis were performed repeatedly. However, despite the intensive treatment, renal failure and neurologic dysfunction progressively worsened, resulting in oliguria and coma. Subsequently adult respiratory distress syndrome was also present. Level of aCL further increased (164.5 $\mathrm{U} / \mathrm{ml})$. Although hemodialysis and respiratory assist with artificial ventilation were performed, the patient died on the 36th day at our hospital. At autopsy, fibrinous material was present over both lungs and petechiae were seen on the surface of the heart, kidneys, and lungs. The brain was severely atrophic (weight $765 \mathrm{~g}$ ), and contained multiple scattered foci of hemorrhagic necrosis. Microscopic examination revealed occlusion of arterioles and capillaries with abundant hyaline thrombi and segmented subendothelial hyaline deposition in multiple or-

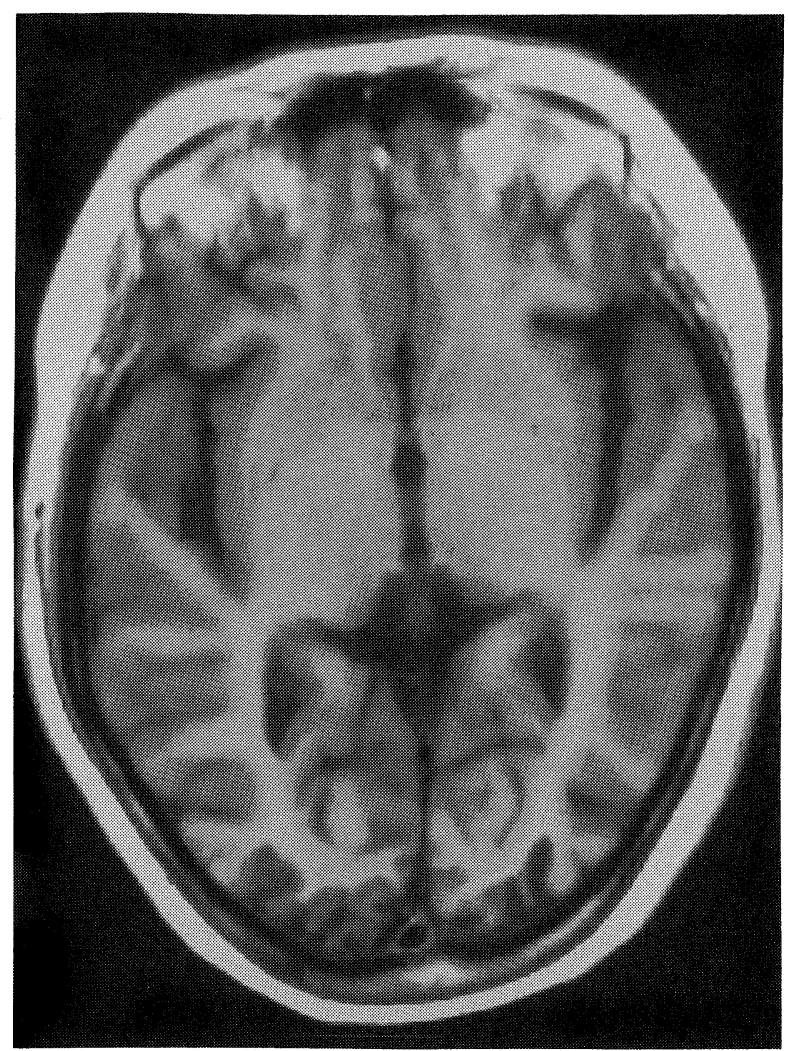

Figure 1. Magnetic resonance imaging of the brain revealed severe global atrophy and ischemic change on bilateral superior frontal gyrus, in addition to infarct area in occipital lobe.

gans including the heart, liver, spleen, pancreas, adrenals, thyroid, kidneys, and brain (Fig. 2A, B).

\section{Discussion}

\section{Catastrophic antiphospholipid syndrome (CAPS)}

The term CAPS was first introduced by Asherson in 1992 to define an accelerated form of APS resulting in multiorgan failure (4). Asherson et al proposed a definition of CAPS that requires involvement of 3 or more organs with thrombosis in the setting of APS (5). CAPS mainly occurs in association with SLE. There is a sudden and explosive onset of multiple vascular occlusions affecting multiple organs such as the lungs, heart, brain, kidney, liver, adrenal grand, and gastrointestinal tract, in various combinations. Treatment of CAPS is not currently standardized and the prognosis is poor with death occurring in $60 \%$ of patients. In one series $(5,6)$, the average age at presentation was 26.1 years with a female-to-male ratio of 2.4 to 1 . Most organs can be involved with pulmonary, cerebral, skin, and renal involvement being most common. Precipitating factors contributing to the development of CAPS include administration of a particular drug, surgical procedures, anticoagulation withdrawal, and some infections, most commonly those affect- 


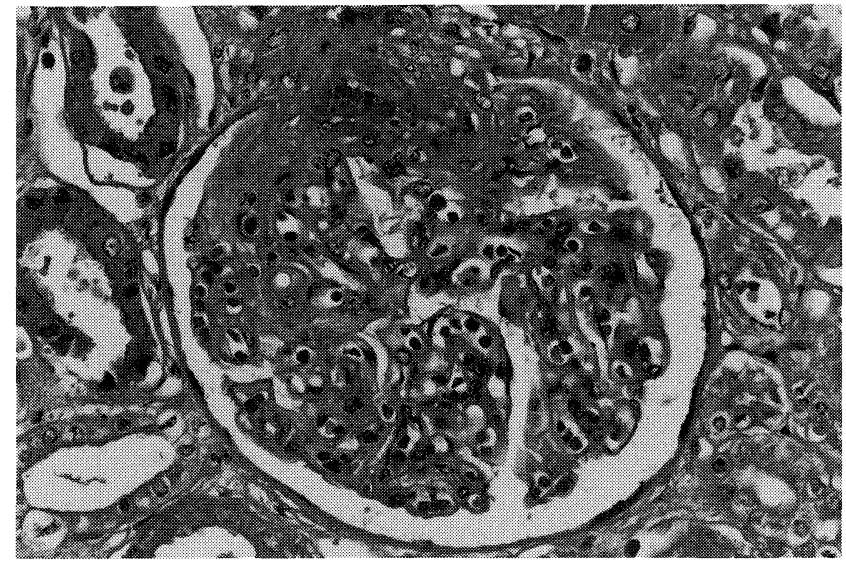

A

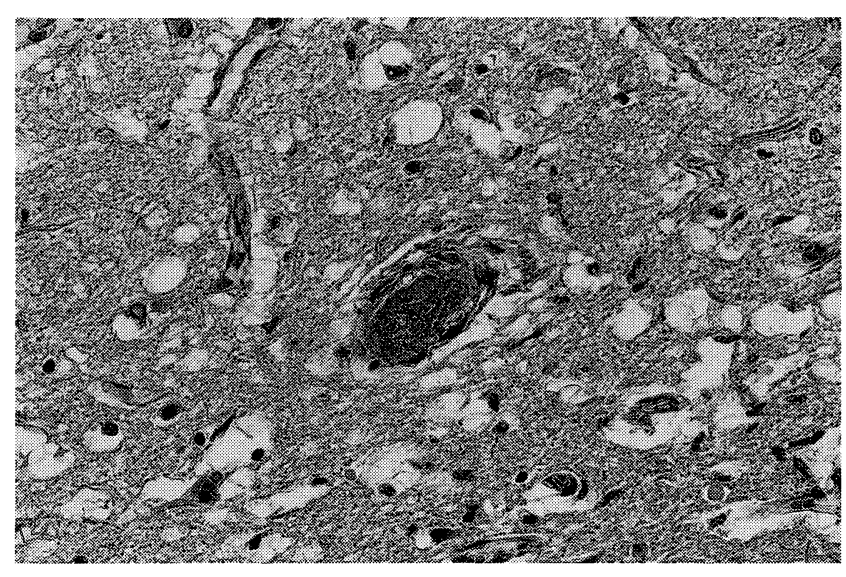

B

Figure 2. At autopsy, microscopic examination revealed occlusion of arterioles and capillaries with abundant hyaline thrombi and segmental subendothelial hyaline deposits both in the kidney (A; HE, stain, $\times 200$ ). In the brain, organized fibrin thrombi, obstruction by intimal proliferation, and recanalization with persistent fibrous webs across arterial lumina were revealed (B; HE, stain, $\times 600)$.

ing the upper respiratory tract. However, in the majority of these patients no such trigger factors are present. Clinically, CAPS mimics DIC and particularly TTP. Recently, it has been proposed that all of these disorders share a common etiology in endothelial cell damage resulting in gross fibrinolytic disturbance (7). Therefore, the differential diagnosis in the three major hypercoagulable states, namely CAPS, DIC, and TTP, is often difficult. For the present patient, we finally diagnosed CAPS in SLE, but she also had several features indicative of TTP.

\section{Differential diagnosis between DIC, TTP, and CAPS}

Our patient was considered to have SLE with high levels of aPL complicated with ITP and AIHA and was thus given a combination of steroids, anticoagulation, and cyclophosphamide. Postmortem examination confirmed predominantly noninflammatory thrombosis and partially coexistent multiorgan vasculitis in the brain, lung, kidney, spleen, pancreas, descending colon, and skin. These findings were compatible with the diagnosis of CAPS in SLE. In SLE, the appearance of multiorgan thrombotic events occurring in rapid chronological sequence after preceding pneumonia would lead to a differential diagnosis between the hypercoagulable states, namely DIC, TTP, and CAPS. As mentioned above, these seemingly separate conditions may present with either essentially the same or a similar clinical picture, therefore the differential diagnosis is difficult and confusing. We analyzed our patient from the clinical and laboratory aspects. First, thrombocytopenia and the generalized noninflammatory thrombosis seen in our patient may be compatible with any of the above three hypercoagulable conditions. However, DIC could be definitively excluded by coagulation tests in our case. Secondary, central nervous system (CNS) manifestations and renal failure with rapid deterioration are characteristic of CAPS. In TTP, CNS manifestation is often transient and either proteinuria or hematuria without serological evidence of renal failure may be the only sign of renal involvement. Thirdly, she demonstrated high levels of aPL, which more increased with her worsened illness. This fact is strongly suggestive of CAPS. We think that the total picture of our patient's features most likely indicates CAPS in SLE.

\section{Thrombogenic mechanisms of CAPS}

CAPS is a relatively new entity and thought to be a rare disease. The exact thrombogenic mechanisms of CAPS remain unclear. Several hypotheses previously implicated direct endothelial damage, platelet activation, inhibition of endogenous anticoagulation factors such as the complex thrombomodulin, protein $\mathrm{C}$, protein $\mathrm{S}$, antithrombin III, prekallikrein, and prostacyclin $(8,9)$. Recently, the pathogenesis of CAPS has been reviewed in some detail, and the role of activated vascular endothelium has been emphasized as an important factor (10). Belmont et al postulated that activation of endothelial cell molecules leads to the expression of membrane-associated coagulation proteins, which could be the targets of aPL (11). The presence of elevated plasma levels of von Willebrand factor (vMF) antigen as markers of endothelial cell damage has been documented in CAPS and DIC, whereas abnormalities of vWF multimers have been reported as the pathogenetic feature of TTP $(12,13)$. In addition, it has been documented that impaired production of thrombomodulin, a thrombin receptor present on the endothelial cell membrane, resulting from endothelial cell damage reduces protein $\mathrm{C}$ activation and plays an important etiological role in intravascular coagulation in CAPS and DIC, as well as in TTP $(14,15)$. Investigation of vWF and thrombomodulin was not performed in the present case.

In addition, as Asherson described, some infections may act as precipitating factors contributing to the development of CAPS (4-7), and pneumonia may have been the trigger in our 


\section{An Autopsy Case Report of CAPS in SLE}

case by somehow causing endothelial damage. Close attention to trigger factors is important for the prevention of CAPS.

In conclusion, CAPS is an uncommon, but life-threatening condition that requires high clinical and pathogenetic awareness. It is hoped that the recent findings regarding the possible pathogenic mechanisms in CAPS may open new avenues to identify trigger factors and to further its treatment and prognosis.

\section{References}

1) Matsuura $Y$, Nawata $Y$, Miike $S$, et al. Lupus anticoagulants in patients with systemic lupus erythematosus. Ryumachi 36: 16-24, 1996.

2) Love PE, Santoro SA. Antiphospholipid antibodies: anticardiolipin and the lupus anticoagulant in systemic lupus erytematosus (SLE) and in nonSLE disorders. Prevalence and clinical significance. Ann Intern Med 112: 682-698, 1990.

3) Harris EN, Baguley E, Asherson RA, et al. Clinical and serological features of the antiphospholipid syndrome (APS). Br J Rheumatol 26: 19, 1987 (Suppl 2).

4) Asherson RA. The catastrophic antiphospholipid syndrome. J Rheumatol 19: 508-512, 1992 (editorial).

5) Asherson RA, Piette J-C. The catastrophic antiphospholipid syndrome 1996: acute multi-organ failure associated with antiphospholipid antibodies: A review of 31 patients. Lupus 5: 414-417, 1996.
6) Asherson RA, Cervera R, Piette JC, et al. The catastrophic antiphospholipid syndrome. Clinical and laboratory features of 50 patients. Medicine 77: 195-207, 1998 (Baltimore).

7) Asherson RA, Cervera R, Font J. Multiorgan thrombotic disorders in systemic lupus erytematosus: a common link?. Lupus 1: 199-203, 1992.

8) Tobelem G, Cariou R, Camez A. The lupus anticoaglant and its role in thrombosis. Blood Rev 1: 21-24, 1987.

9) Khamashta MA, Harris EN, Gharavi AE, et al. Immune mediated mechanism for thrombosis; antiphospholipid antibody binding to platelet membranes. Ann Rheum Dis 47: 849-854, 1988.

10) Golden BD, Belmont HM. The role of microvasculopathy in the catastrophic antiphospholipid syndrome: comment on the article by Neuwelt et al. Arthritis Rheum 41: 751-752, 1998 (letter; comment).

11) Belmont HM, Abramson SB, Lie JT. Pathology and pathogenesis of vascular injury in systemic lupus erythematosis. Arthritis Rheum 39: 9-22, 1996.

12) Furlan M, Robles R, Galbusera $M$, et al. von Willebrand factor-cleaving protease in thrombotic thrombocytopenic purpura and the hemolytic-uremic syndrome. N Engl J Med 339: 1578-1584, 1998 (see comment).

13) Holt CM, Lindsey N, Moult J, et al. Antibody-dependent cellular cytotoxicity of vascular endothelium: Characterization and pathogenic associations in systemic sclerosis.Clin Exp Immunol 78: 359-365, 1989.

14) Karmochkine M, Boffa MC, Piette JC, et al. Increase in plasma thrombomodulin in lupus erythematosus with antiphopholipid antibodies. Blood 79: 837-838, 1992 (letter).

15) Takahashi $\mathrm{H}$, Hanano $\mathrm{N}$, Wada $\mathrm{K}$, et al. Circulating thrombomodulin in thrombotic thrombocytopenic purpura. Am J Haematol 38: 174-177, 1991. 